\title{
On finite generation of Noetherian algebras over two-dimensional regular local rings
}

\author{
Amartya Kumar Dutta ${ }^{\star}$, Neena Gupta ${ }^{\star \star}$ and Nobuharu Onoda ${ }^{\dagger *}$

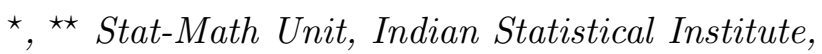 \\ 203 B.T. Road, Kolkata 700 108, India. \\ e-mail : amartya.28@gmail.com and neenag@isical.ac.in \\ ${ }^{\dagger}$ Department of Mathematics, University of Fukui, \\ Bunkyo 3-9-1, Fukui 910-8507, Japan \\ e-mail : onoda@u-fukui.ac.jp
}

April 21, 2020

\begin{abstract}
Let $R$ be a complete regular local ring with an algebraically closed residue field and let $A$ be a Noetherian $R$-subalgebra of the polynomial ring $R[X]$. It has been shown in [4] that if $\operatorname{dim} R=1$, then $A$ is necessarily finitely generated over $R$. In this paper, we give necessary and sufficient conditions for $A$ to be finitely generated over $R$ when $\operatorname{dim} R=2$ and present an example of a Noetherian normal non-finitely generated $R$-subalgebra of $R[X]$ over $R=\mathbb{C}[[u, v]]$.
\end{abstract}

Keywords Finite generation, subalgebra of polynomial algebra, dimension formula, Nagata ring, complete local ring, regular local ring, Krull domain, excellent ring.

2010 MSC. Primary: 13E15, Secondary: 13F20, 13J10, 13H05

\section{Introduction}

Let $R$ be a Noetherian ring and $A$ a Noetherian $R$-subalgebra of $R[X]$, where $R[X]$ is the polynomial ring in one indeterminate $X$ over $R$. Then $A$ need not be a finitely generated $R$ algebra, in general. In fact, by an example of Eakin [5, p. 79], even when $R$ is the polynomial ring $\mathbb{C}[t]$, there exist non-finitely generated Noetherian rings $A$ satisfying $R \subseteq A \subseteq R[X]$. However, it has been shown in [4] that when $R$ is a complete discrete valuation ring with an algebraically closed residue field (e.g., when $R=\mathbb{C}[[t]]$ ) then any Noetherian $R$-subalgebra $A$ of $R[X]$ must be finitely generated. A precise version of the result is quoted below ([4, Theorem 4.2]).

\footnotetext{
${ }^{*}$ Present address: Fukui Study Center, The Open University of Japan, AOSSA 7F, Teyose 1-4-1, Fukui 910-0858, Japan
} 
Theorem 1.1. Let $(R, \pi)$ be a complete discrete valuation ring with residue field $k$ and field of fractions $K$. Suppose that the algebraic closure $\bar{k}$ of $k$ is a finite extension of $k$. Let $A$ be a Noetherian domain containing $R$ such that $A\left[\pi^{-1}\right]$ is a finitely generated $K$-algebra and $\operatorname{tr} \cdot \operatorname{deg}_{R} A=1$. Then $A$ is finitely generated over $R$. In particular, if $A$ is a Noetherian $R$-subalgebra of $R[X]$, then $A$ is finitely generated over $R$.

Over a complete discrete valuation ring, the following result ([4, Proposition 3.4]) relates the finite generation of a Noetherian normal $R$-subalgebra of $R[X]$ with the transcendence degree of certain fibres.

Theorem 1.2. Let $(R, \pi)$ be a complete discrete valuation ring with residue field $k$ and field of fractions $K$. Let $A$ be a Krull domain such that $R \subseteq A, A\left[\pi^{-1}\right]$ is a finitely generated $K$-algebra and tr. $\operatorname{deg}_{R} A=1$. Then $A$ is finitely generated over $R$ if tr.deg $\operatorname{deg}_{k} A / P>0$ for each associated prime ideal $P$ of $\pi A$.

In this paper, we explore two-dimensional analogues of the above results. Note that the integral domain $A$ is flat over the discrete valuation ring $R$ in Theorem 1.1 and this leads us to the following question:

Question 1.3. Let $R$ be a complete regular local ring of dimension two with algebraically closed residue field. Let $A$ be a Noetherian $R$-subalgebra of $R[X]$. Is $A$ finitely generated over $R$, say when $A$ is normal and flat over $R$ ?

Recall that if the ring $A(\neq R)$ in the above question is factorial, then $A \cong_{R} R[X]$ by a result of Abhyankar-Eakin-Heinzer [1, Theorem 4.1].

Example 4.4, the main example of this paper, provides a counter example to the above question. However, our main theorem (Theorem 3.6) shows that under an additional fibre condition on $A$ (similar to the criterion in Theorem 1.2), we do have a two-dimensional analogue of Theorem 1.1. We quote a consequence of our main result (cf. Theorem 3.6 and Corollary 3.8):

Theorem I. Let $(R, m)$ be a complete two-dimensional regular local ring with residue field $k$. Suppose that the algebraic closure $\bar{k}$ of $k$ is a finite extension of $k$. Let $A$ be a Noetherian domain such that $A$ is a flat $R$-algebra with $\operatorname{tr} \cdot \operatorname{deg}_{R} A=1$. Suppose that there exists $\pi \in m$ such that $A\left[\pi^{-1}\right]$ is a finitely generated $R\left[\pi^{-1}\right]$-algebra and $\operatorname{tr} \cdot \operatorname{deg}_{R /(P \cap R)} A / P>0$ for each $P \in \operatorname{Ass}_{A}(A / \pi A)$. Then $A$ is finitely generated over $R$.

Regarding Question 1.3, it has been shown in [4, Lemma 3.3] that over a one-dimensional Noetherian domain $R$, any Krull domain which is an $R$-subalgebra of $R[X]$ is Noetherian. We give an example (Example 4.6) to show that this result does not hold when $\operatorname{dim} R>1$, not even when $R$ is a complete regular local domain with an algebraically closed residue field.

We now give a layout of the paper. In Section 2, we recall a few results which we shall use to prove our statements. In Section 3, as a step towards Theorem I, we first prove generalisations of Theorems 1.1 and 1.2 (Propositions 3.2 and 3.1). Then we consider the case where the base ring $R$ is a two-dimensional Noetherian local domain, and establish a few criteria, including Theorem I, for Noetherian property and finite generation of $R$-subalgebras of a finitely generated $R$-algebra. We also give a sufficient condition for the ring $A$ of Theorem 
I to be Noetherian under some fibre conditions on $A$ (Theorem 3.14). In Section 4, we demonstrate our examples. These examples are based on the methodology of Lemmas 4.14.3. In Appendix, we establish a condition for finite generation of algebras over excellent rings. This result was earlier established by the third author in [11], over fields and the proof is essentially the same.

\section{Preliminaries}

Throughout the paper $R$ will denote a commutative ring with unity. The notation $A=R^{[n]}$ will denote that $A$ is a polynomial ring in $n$ variables over $R$. For an element $c$ in $A$, the notation $A_{c}$ will denote the ring $T^{-1} A$, where $T$ is the multiplicatively closed set $\left\{c^{n} \mid n \geq 0\right\}$.

Definition. A Noetherian ring $R$ is said to be a Nagata ring (or a pseudo-geometric ring) if, for every prime ideal $p$ of $R$ and for every finite algebraic extension field $L$ of the field of fractions $k(p)$ of $R / p$, the integral closure of $R / p$ in $L$ is a finite module over $R / p$.

Any Noetherian complete local ring is a Nagata ring ([8, p. 234, Corollary 2]) and any finitely generated algebra over a Nagata ring is a Nagata ring ([8, p. 240, Theorem 72]).

We first recall the following version of dimension inequality (cf. [3, Theorem 2]

Theorem 2.1. Let $R$ be a Noetherian integral domain and $B$ an integral domain containing $R$. Let $P$ be a prime ideal of $B$ and $p=P \cap R$. Then

$$
\text { ht } P+\text { tr. } \operatorname{deg}_{R / p} B / P \leq \text { ht } p+\text { tr. } \operatorname{deg}_{R} B .
$$

Let the notation and assumptions be the same as in Theorem 2.1. Then we say that $P$ satisfies the dimension equality relative to $R$ if the equality holds in (2.1), and we say that the dimension formula holds between $R$ and $B$ if every prime ideal $P$ in $B$ satisfies the dimension equality relative to $R$. It is known that if $R$ is universally catenary and $B$ is finitely generated over $R$, then the dimension formula holds between $R$ and $B$ (cf. [9, Theorem 15.6]). For later use we note the following.

Lemma 2.2. Let $R \subseteq B$ be integral domains such that $R$ is Noetherian. Let $P$ be a prime ideal in $B$ and let $p=P \cap R$. Suppose that ht $p=1$. Then $\operatorname{tr} \cdot \operatorname{deg}_{R / p} B / P \leq \operatorname{tr} \cdot \operatorname{deg}_{R} B$, where the equality holds if and only if ht $P=1$ and $P$ satisfies the dimension equality relative to $R$. In particular, the equality holds if ht $P=1, R$ is universally catenary, and $B$ is finitely generated over $R$.

Proof. Since ht $p=1$, we have

$$
\text { tr. } \operatorname{deg}_{R / p} B / P \leq 1-\text { ht } P+\text { tr. } \operatorname{deg}_{R} B \leq \text { tr. } \operatorname{deg}_{R} B
$$

by Theorem 2.1. From this it follows that $\operatorname{tr} \cdot \operatorname{deg}_{R / p} B / P \leq \operatorname{tr} \cdot \operatorname{deg}_{R} B$, and the equality holds if and only if ht $P=1$ and $P$ satisfies the dimension equality relative to $R$.

For convenience, we now quote a few other known results which will be needed in our arguments. We first state an easy lemma. 
Lemma 2.3. Let $B \subseteq A$ be integral domains. Suppose that there exists a nonzero element $t$ in $B$ such that $B\left[t^{-1}\right]=A\left[t^{-1}\right]$ and $t A \cap B=t B$. Then $B=A$. In particular, if $t$ is a nonzero prime element in $B$, then (by letting $A=B\left[t^{-1}\right] \cap B_{(t B)}$ ), we have $B=B\left[t^{-1}\right] \cap B_{(t B)}$.

The following result, giving a criterion for an integral domain to be Noetherian, is proved in [4, Lemma 2.8].

Lemma 2.4. Let $D$ be an integral domain. Suppose that there exists a nonzero element $t$ in $D$ such that

(I) $D\left[t^{-1}\right]$ is a Noetherian ring.

(II) $t D$ is a maximal ideal of $D$.

(III) ht $(t D)=1$ (or, equivalently, $\bigcap_{n \geq 1} t^{n} D=(0)$ ).

Then $D$ is a Noetherian ring.

For ready reference, we state below a comaximality criterion for a ring to be Noetherian, or an algebra to be finitely generated, which can be proved easily.

Lemma 2.5. Let $a$ and $b$ be two regular elements of a ring $B$ such that $(a, b) B=B$. Then the following statements hold.

(1) If $B_{a}$ and $B_{b}$ are Noetherian, then $B$ is Noetherian.

(2) If $B$ is an $R$-algebra such that $B_{a}$ and $B_{b}$ are finitely generated $R$-algebras, then $B$ is a finitely generated $R$-algebra.

We state below another elementary result on finite generation ([13, p. 201]).

Lemma 2.6. Let $R$ be a Noetherian domain and $B$ an $R$-subalgebra of a finitely generated $R$-algebra $C$. If $C$ is integral over $B$, then $B$ is finitely generated over $R$.

For a proof of the following result on finite generation, see [7, 2.1] or [10, Proposition 2.11].

Proposition 2.7. Let $R$ be a Noetherian domain and $A$ a subalgebra of a finitely generated $R$-algebra. Then there exists a nonzero element $f$ in $A$ such that $A\left[f^{-1}\right]$ is a finitely generated $R$-algebra.

We recall below the local-global result [10, Theorem 2.20] which reduces the question of finite generation of a subalgebra of a polynomial algebra to the local situation. Recall that an integral domain $C$ containing $R$ is said to be a locality (or essentially of finite type) over $R$ if there exists a finitely generated $R$-algebra $B$ and a prime ideal $Q$ of $B$ such that $C=B_{Q}$.

Theorem 2.8. Let $R$ be a Noetherian domain and $B$ an integral domain containing $R$ such that there exists a nonzero $f \in B$ for which $B\left[f^{-1}\right]$ is a finitely generated $R$-algebra. Then the following statements hold. 
(1) If $B_{M}$ is a locality over $R$ for every maximal ideal $M$ of $B$, then $B$ is a finitely generated $R$-algebra.

(2) If $B_{m}$ is a finitely generated $R_{m}$-algebra for each maximal ideal $m$ of $R$, then $B$ is a finitely generated $R$-algebra.

We now state a criterion for a torsion-free module over a two-dimensional regular local ring to be flat. For the lack of a ready reference, we give below a proof.

Lemma 2.9. Let $R$ be a two-dimensional regular local ring and $\left\{\pi_{1}, \pi_{2}\right\}$ a regular system of parameters in $R$. Let $M$ be a torsion-free $R$-module such that $\pi_{2}$ is $\left(M / \pi_{1} M\right)$-regular. Then $M$ is flat over $R$.

Proof. By [2, Lemma 4.1], it suffices to show that for every prime ideal $P$ of $R$, the natural map

$$
\phi: P \otimes_{R} M \longrightarrow M
$$

is injective.

Fix a prime ideal $P$ of $R$. Suppose $\operatorname{ht}(P)=0$. Then $P=0$ as $R$ is an integral domain and hence the natural map $\phi$ in (2.2) is trivially injective.

Next, suppose that $\operatorname{ht}(P)=1$. Since $R$ is a regular local ring and hence a UFD, $P$ is a principal ideal. Since $M$ is torsion-free, it then follows that the natural map $\phi$ in (2.2) is injective.

Finally, we consider the case $h t(P)=2$, i.e., $P=\left(\pi_{1}, \pi_{2}\right) R$, the unique maximal ideal of $R$. Then, any element $\xi$ in $P \otimes_{R} M$ can be expressed as

$$
\xi=\pi_{1} \otimes m_{1}+\pi_{2} \otimes m_{2}
$$

for some $m_{1}, m_{2} \in M$. Suppose that $\phi(\xi)=0$, i.e.,

$$
\pi_{1} m_{1}+\pi_{2} m_{2}=0 \text {. }
$$

Since $\pi_{2}$ is $\left(M / \pi_{1} M\right)$-regular, we have $m_{2}=\pi_{1} m$ for some $m \in M$. Since $M$ is torsionfree, from (2.4), we have $m_{1}=-\pi_{2} m$. Substituting in (2.3), we see that $\xi=0$, i.e., $\phi$ is injective.

\section{Main Results}

In this section we shall prove our main theorem and record a few auxiliary observations.

For the proof of our main theorem, we first record two results on finite generation over onedimensional Nagata domains, which are generalisations of Theorems 1.2 and 1.1, respectively.

Proposition 3.1. Let $R$ be a one-dimensional Nagata domain, and let $B$ be a Krull domain such that $R \subseteq B$ with tr. $\operatorname{deg}_{R} B=1$. Suppose that there exists $\pi \in R$ such that $B\left[\pi^{-1}\right]$ is a finitely generated $R\left[\pi^{-1}\right]$-algebra. Then the following statements are equivalent.

(i) $B$ is a finitely generated $R$-algebra. 
(ii) tr. $\operatorname{deg}_{R / P \cap R} B / P>0$ for every minimal prime ideal $P$ of $\pi B$.

Proof. (i) $\Rightarrow$ (ii). Let $P$ be a minimal prime ideal of $\pi B$. Then ht $P=1$ as $B$ is a Krull domain and $\operatorname{ht}(P \cap R)=1$ as $\operatorname{dim}(R)=1$ and $P \cap R \neq 0$. By [9, p. 255, Corollary 2], $R$ is universally catenary. Hence, by the dimension formula Lemma 2.2, we have $\operatorname{tr} \cdot \operatorname{deg}_{R / P \cap R} B / P=1$.

(ii) $\Rightarrow$ (i). Let $\bar{R}$ denote the normalisation of $R$. Then $\bar{R} \subseteq B$, and $\bar{R}$ is a finite $R$-module because $R$ is a Nagata domain. Hence it is enough to show that $B$ is a finitely generated $\bar{R}$-algebra.

If $P$ is a minimal prime ideal of $\pi B, p=P \cap R$ and $p^{\prime}=P \cap \bar{R}$, then $\bar{R} / p^{\prime}$ is integral over $R / p$, so that tr. $\operatorname{deg}_{\bar{R} / p^{\prime}} B / P=\operatorname{tr} \cdot \operatorname{deg}_{R / p} B / P>0$. Thus, replacing $R$ by $\bar{R}$, we assume that $R$ is a Dedekind Nagata domain.

Since $B\left[\pi^{-1}\right]$ is a finitely generated $R\left[\pi^{-1}\right]$-algebra, by Theorem $2.8(2)$, it suffices to show that $B_{m}\left(=B \otimes_{R} R_{m}\right)$ is a finitely generated $R_{m}$-algebra for every maximal ideal $m$ of $R$ with $\pi \in m$.

Fix a maximal ideal $m$ of $R$ containing $\pi$ and set $k:=R / m=R_{m} / m R_{m}$. Now note that for any $P \in \operatorname{Spec} B$, if $P B_{m}$ is a minimal prime ideal of $\pi B_{m}$, then $P$ is a minimal prime ideal of $\pi B$. Moreover, $P B_{m} \cap R_{m}=m R_{m}$ and, from our hypothesis, it follows that $\operatorname{tr} \cdot \operatorname{deg}_{k} B_{m} / P B_{m}>0$. Thus, replacing $R$ by $R_{m}$, we may further assume that $R$ is a discrete valuation ring which is also a Nagata ring.

The desired result now follows from [4, Proposition 3.4].

Proposition 3.2. Let $(R, m)$ be a complete one-dimensional Noetherian local domain with residue field $k$ and field of fractions $K$. Suppose that the algebraic closure $\bar{k}$ of $k$ is a finite extension of $k$. Let $B$ be a Noetherian integral domain such that $R \subseteq B, \operatorname{tr} \cdot \operatorname{deg}_{R} B \leq 1$, and $B \otimes_{R} K$ is a finitely generated $K$-algebra. Then $B$ is a finitely generated $R$-algebra.

Proof. We note that $\operatorname{dim} B \leq 2$. Indeed, if $P$ is a prime ideal of $B$, then setting $p:=P \cap R$, we have ht $p \leq 1$, so that, by Theorem 2.1 ,

$$
\text { ht } P \leq \text { ht } p+\text { tr. } \cdot \operatorname{deg}_{R} B-\operatorname{tr} \cdot \operatorname{deg}_{R / p} B / P \leq 2,
$$

as claimed. Let $\bar{R}$ be the normalisation of $R$ and $\bar{B}$ the normalisation of $B$. Then $\bar{R}$ is a complete discrete valuation ring which is a finite $R$-module (cf. [9, p. 263]). Let $k^{\prime}$ denote the residue field of $\bar{R}$. Then $k^{\prime}$ is algebraic over $k$ and hence $\bar{k}$ is a finite algebraic extension of $k^{\prime}$. Since $\operatorname{dim} B \leq 2, \bar{B}$ is a Noetherian domain (cf. [12, Theorems 33.2, 33.12]). Note that $K=R\left[\pi^{-1}\right]$ for any $\pi(\neq 0) \in m$. Since $\bar{R} \subseteq \bar{B}$, $\operatorname{tr} \cdot \operatorname{deg}_{\bar{R}} \bar{B} \leq 1$ and $\bar{B} \otimes_{\bar{R}} K$ is a finitely generated $K$-algebra, it follows from [4, Theorem 4.2] that $\bar{B}$ is a finitely generated $\bar{R}$-algebra. Hence $\bar{B}$ is a finitely generated $R$-algebra, because $\bar{R}$ is a finite $R$-module. Since $\bar{B}$ is integral over $B, B$ is a finitely generated $R$-algebra by Lemma 2.6.

We also need the following technical result in the proof of our main theorem.

Proposition 3.3. Let $(R, m)$ be a two-dimensional Nagata local domain with residue field $k$, and let $A$ be an integral domain such that $R \subseteq A$ with $\operatorname{tr} \cdot \operatorname{deg}_{R} A=1$. For $P \in \operatorname{Spec} A$, let $p$ denote $P \cap R$. Set $\Delta:=\left\{P \in \operatorname{Ass}_{A}(A / \pi A) \mid\right.$ ht $\left.p=1\right\}$. Suppose that $A$ satisfies the following hypotheses. 
(I) A is either a Krull domain or a Noetherian domain.

(II) There exists a nonzero element $\pi \in m$ such that $A\left[\pi^{-1}\right]$ is finitely generated over $R\left[\pi^{-1}\right]$.

(III) tr. $\operatorname{deg}_{R / p} A / P>0$ for every $P \in \Delta$.

Then the following statements hold.

(1) $A\left[f^{-1}\right]$ is finitely generated over $R\left[f^{-1}\right]$ for every nonzero $f \in m$.

(2) Every $P \in \Delta$ has the following properties.

(a) tr.deg $\operatorname{deg}_{R / p} A / P=1$.

(b) $P$ satisfies the dimension equality relative to $R$.

(c) $A / P \otimes_{R / p} k(p)$ is finitely generated over $k(p)$, where $k(p)=R_{p} / p R_{p}$ is the field of fractions of $R / p$.

Proof. (1) Fix $f(\neq 0) \in m$. Set $S:=R\left[f^{-1}\right]$ and $C:=A\left[f^{-1}\right]$. Note that $S$ is a onedimensional Nagata domain. Also note that $A\left[\pi^{-1}\right]$ is a Nagata domain because $A\left[\pi^{-1}\right]$ is finitely generated over $R\left[\pi^{-1}\right]$ and $R\left[\pi^{-1}\right]$ is a Nagata domain.

Now, if $f \in \sqrt{\pi A}$, then $f^{n}=\pi a$ for some $n>0$ and $a \in A$, so that $C=A\left[f^{-n}\right]=$ $A\left[\pi^{-1}, a^{-1}\right]$ is finitely generated over $S$ and we are through. So we assume that $f \notin \sqrt{\pi A}$, i.e., $\pi C$ is a proper ideal of $C$.

Case (i). First, we consider the case where $A$ is a Krull domain. In this case, $C$ is a Krull domain such that $C\left[\pi^{-1}\right]$ is finitely generated over $S\left[\pi^{-1}\right]$. Let $Q$ be a minimal prime ideal of $\pi C$. Set $P:=Q \cap A$ and $\mathfrak{q}:=P \cap S=Q \cap S$. Then $P$ is a minimal prime ideal of $\pi A$ and $p=P \cap R=\mathfrak{q} \cap R$. Note that ht $\mathfrak{q}=1$ as $\operatorname{dim} S=1$, and hence, ht $p=1$. Therefore, tr. $\operatorname{deg}_{S / \mathfrak{q}} C / Q\left(=\operatorname{tr} \cdot \operatorname{deg}_{R / p} A / P\right)>0$, by our hypothesis. Hence, $C$ is finitely generated over $S$ by Proposition 3.1 .

Case (ii). Next, we consider the case where $A$ is a Noetherian domain. Let $D$ denote the normalisation of $A$. Then $D$ is a Krull domain (cf. [12, Theorem 33.10]). We shall verify that $D$ too satisfies all the hypotheses given for $A$.

Since $D\left[\pi^{-1}\right]$ is the normalisation of the Nagata domain $A\left[\pi^{-1}\right]$, it follows that $D\left[\pi^{-1}\right]$ is a finite $A\left[\pi^{-1}\right]$-module, and hence $D\left[\pi^{-1}\right]$ is finitely generated over $R\left[\pi^{-1}\right]$.

Let $Q$ be a minimal prime ideal of $\pi D$ such that ht $(Q \cap R)=1$. Let $P=Q \cap A$. Then $p=P \cap R=Q \cap R$. By [12, Theorem 33.11], $P$ is an associated prime ideal of $\pi A$ so that tr. $\operatorname{deg}_{R / p} A / P>0$ by our hypothesis. Since $D / Q$ is integral over $A / P$, it follows that tr. $\operatorname{deg}_{R / p} D / Q>0$.

Thus, by Case (i), $D\left[f^{-1}\right]$ is finitely generated over $R\left[f^{-1}\right]$. Since $D\left[f^{-1}\right]$ is integral over $A\left[f^{-1}\right]$, we conclude, by Lemma $\left[2.6\right.$, that $A\left[f^{-1}\right]$ is finitely generated over $R\left[f^{-1}\right]$.

(2) Fix $P \in \Delta$.

(2a) Since ht $p=1$, we have tr. $\operatorname{deg}_{R / p} A / P \leq \operatorname{tr} \cdot \operatorname{deg}_{R} A$ by Lemma 2.2. But, by our hypotheses, tr. $\operatorname{deg}_{R} A=1$ and tr. $\operatorname{deg}_{R / p} A / P \geq 1$. Thus, tr. $\operatorname{deg}_{R / p} A / P=\operatorname{tr} \cdot \operatorname{deg}_{R} A=1$. 
(2b) Since the equality tr. $\operatorname{deg}_{R / p} A / P=\operatorname{tr} \cdot \operatorname{deg}_{R} A$ holds, $P$ satisfies the dimension equality relative to $R$ again by Lemma 2.2 .

(2c) Set $R^{\prime}:=R / p$ and $B:=A / P$. Then $R^{\prime}$ is a one-dimensional local domain. Hence, for any $f(\neq 0) \in m \backslash p$, we have $R^{\prime}\left[f^{-1}\right]=k(p)$, so that $B \otimes_{R^{\prime}} k(p)=A\left[f^{-1}\right] / P A\left[f^{-1}\right]$. Thus, the assertion is an immediate consequence of (1).

We have the following consequence for the case $R$ is complete and the residue field of $R$ is an algebraically closed field or a real closed field.

Corollary 3.4. Let $(R, m)$ be a two-dimensional complete local domain with residue field $k$ such that $[\bar{k}: k]<\infty$, where $\bar{k}$ denotes the algebraic closure of $k$. Let $A$ be an integral domain satisfying all the hypotheses of Proposition 3.3. Let $\Delta$ be as in Proposition 3.3. Suppose that $A / P$ is Noetherian for some $P \in \Delta$. Then $A / P$ is finitely generated over $R / p$, where $p=P \cap R$ as in Proposition 3.3 .

Proof. Since $R / p$ is a one-dimensional complete local domain with residue field $k$, the assertion follows from Proposition 3.3(2) and Proposition 3.2.

We also need the following easy lemma.

Lemma 3.5. Let $R$ be a Noetherian domain and $A$ a Krull domain containing $R$. Let $\pi$ be a nonzero element in $A$ with $\pi A \neq A$. Then the following assertions hold.

(1) If $A / P$ is finitely generated over $R$ for every $P \in \operatorname{Ass}_{A}(A / \pi A)$, then $A / \pi A$ is finitely generated over $R$.

(2) If $A / P$ is a finite $R$-module for every $P \in \operatorname{Ass}_{A}(A / \pi A)$, then $A / \pi A$ is a finite $R$-module.

Proof. Let $\operatorname{Ass}_{A}(A / \pi A)=\left\{P_{1}, \ldots, P_{n}\right\}$, and let $\pi A=P_{1}^{\left(e_{1}\right)} \cap P_{2}{ }^{\left(e_{2}\right)} \cap \cdots \cap P_{n}{ }^{\left(e_{n}\right)}$ be the primary decomposition of $\pi A$. Then

$$
A / \pi A \hookrightarrow B:=A / P_{1}^{\left(e_{1}\right)} \times A / P_{2}^{\left(e_{2}\right)} \times \cdots \times A / P_{n}{ }^{\left(e_{n}\right)}
$$

is a finite integral extension. Hence if $B$ is finitely generated over $R$, then so is $A / \pi A$ by Lemma 2.6, and if $B$ is a finite $R$-module, then so is $A / \pi A$ as $R$ is Noetherian.

Now, let $P \in \operatorname{Ass}_{A}(A / \pi A)$ and let $e$ be a positive integer. Note that in both the cases (1) and (2), $A / P$ is Noetherian, and hence so is $A / P^{(e)}$ by [9, Theorem 12.7]. Note also that, setting $C:=A / P^{(e)}$, we have $C / \sqrt{(0)}=A / P$. Therefore if $A / P$ is finitely generated over $R$ (resp. a finite $R$-module), then $A / P^{(e)}$ is also finitely generated over $R$ (resp. a finite $R$-module) (cf. [4, Lemma 4.1]). Thus the above $\operatorname{ring} B$ in (3.1) is finitely generated over $R$ for the case (1), and is a finite $R$-module for the case (2). This completes the proof.

We are now ready to prove our main theorem.

Theorem 3.6. Let $(R, m)$ be a complete two-dimensional Noetherian local domain whose residue field $k$ satisfies the condition $[\bar{k}: k]<\infty$, where $\bar{k}$ is the algebraic closure of $k$. Let $A$ be a Krull domain such that $R \subseteq A$ with $\operatorname{tr} \cdot \operatorname{deg}_{R} A=1$. Suppose $A$ satisfies the following hypotheses. 
(I) There exists a nonzero element $\pi \in m$ such that $A\left[\pi^{-1}\right]$ is finitely generated over $R\left[\pi^{-1}\right]$.

(II) $\operatorname{ht}(P \cap R)=1$ for every $P \in \operatorname{Ass}_{A}(A / \pi A)$.

Then the following conditions are equivalent.

(i) A is finitely generated over $R$.

(ii) $A$ is Noetherian and tr. $\operatorname{deg}_{R / p} A / P>0$ for each $P \in \operatorname{Ass}_{A}(A / \pi A)$.

(iii) $A / \pi A$ is Noetherian and tr. $\operatorname{deg}_{R / p} A / P>0$ for each $P \in \operatorname{Ass}_{A}(A / \pi A)$.

(iv) $A / P$ is Noetherian and tr.deg $\operatorname{deg}_{R / p} A / P>0$ for each $P \in \operatorname{Ass}_{A}(A / \pi A)$.

Proof. (i) $\Rightarrow$ (ii). $A$ is Noetherian by Hilbert Basis Theorem. Let $P \in \operatorname{Ass}_{A}(A / \pi A)$ and set $p:=P \cap R$. Then ht $P=1$ because $A$ is Krull. Therefore, since the complete local ring $R$ is universally catenary (cf. [9, Theorem 29.4(ii)]) and $A$ is finitely generated over $R$, by Lemma 2.2, we have tr. $\operatorname{deg}_{R / p} A / P=\operatorname{tr} \cdot \operatorname{deg}_{R} A=1$.

(ii) $\Rightarrow$ (iii) and (iii) $\Rightarrow$ (iv) are trivial.

(iv) $\Rightarrow$ (i). Note that the Noetherian complete local ring $R$ is an excellent local ring ([8, p. 260, 34.B]). We now verify that the element $\pi$ in the Krull domain $A$ satisfies all the hypotheses of Proposition 5.1 in Section 5.

By hypothesis (I), $A\left[\pi^{-1}\right]$ is a finitely generated $R$-algebra.

The hypothesis (II) and the conditions in (iv) show, by Corollary 3.4, that $A / P$ is finitely generated over $R /(P \cap R)$ for every $P \in \operatorname{Ass}_{A}(A / \pi A)$. Therefore, by Lemma 3.5, $A / \pi A$ is finitely generated over $R$.

Finally, let $P$ be a minimal prime ideal of $\pi A$. Then ht $P=1$ as $A$ is a Krull domain. Also, by Proposition $3.3(2 \mathrm{~b}), P$ satisfies the dimension equality relative to $R$.

Therefore, applying Proposition 5.1, we conclude that $A$ is finitely generated over $R$.

Corollary 3.7. Let $(R, m)$ and $k$ be as in Theorem 3.6 and let $A$ be a Noetherian domain such that $R \subseteq A$ with tr. $\operatorname{deg}_{R} A=1$. Suppose A satisfies the following hypotheses.

(I) There exists a nonzero element $\pi \in m$ such that $A\left[\pi^{-1}\right]$ is finitely generated over $R\left[\pi^{-1}\right]$.

(II) $\operatorname{ht}(P \cap R)=1$ and tr. $\operatorname{deg}_{R /(P \cap R)} A / P>0$ for every $P \in \operatorname{Ass}_{A}(A / \pi A)$.

Then $A$ is finitely generated over $R$.

Proof. Let $D$ be the normalisation of $A . D$ is a Krull domain by [12, Theorem 33.10]. Since $D$ is integral over $A$, by Lemma 2.6, it suffices to show that $D$ is finitely generated over $R$. We verify that the Krull domain $D$ satisfies all the general hypotheses and condition (iv) of Theorem 3.6. Let $Q \in \operatorname{Ass}_{D}(D / \pi D), P=Q \cap A$ and $p=Q \cap R=P \cap R$. We will show:

(a) ht $p=1$.

(b) For every $f \in m \backslash p, D\left[f^{-1}\right]$ is finitely generated over $R\left[f^{-1}\right]$.

(c) $D / Q$ is Noetherian. 
(d) tr. $\operatorname{deg}_{R / p} D / Q>0$.

(a) By [12, Theorem 33.10], $P \in \operatorname{Ass}_{A}(A / \pi A)$ and hence ht $p=1$ by hypothesis (II).

(b) Fix $f \in m \backslash p$. By Proposition $3.3(1), A\left[f^{-1}\right]$ is finitely generated over the Nagata ring $R\left[f^{-1}\right]$ and hence $A\left[f^{-1}\right]$ is also a Nagata ring. Thus $D\left[f^{-1}\right]$, being the normalisation of the Nagata ring $A\left[f^{-1}\right]$, is a finite $A\left[f^{-1}\right]$-module. Hence, $D\left[f^{-1}\right]$ is finitely generated over $R\left[f^{-1}\right]$.

(c) By Corollary 3.4, $A / P$ is finitely generated over the Nagata ring $R$ and hence $A / P$ is a Nagata ring. Since $D\left[f^{-1}\right.$ is a finite $A\left[f^{-1}\right]$-module, it follows that the field of fractions of $D / Q$ is a finite extension of the field of fractions of $A / P$. Since $D / Q$ is integral over the Nagata ring $A / P$, it then follows that $D / Q$ is a finite $A / P$-module; in particular $D / Q$ is Noetherian.

(d) Since $D / Q$ is a finite $A / P$-module, tr. $\operatorname{deg}_{R / p} D / Q=\operatorname{tr} \cdot \operatorname{deg}_{R /(P \cap R)} A / P>0$ by hypothesis (II).

Thus, $D$ is finitely generated over $R$ by Theorem 3.6 and hence $A$ is finitely generated over $R$ by Lemma 2.6 .

In the next result, we will see that the hypothesis "ht $(P \cap R)=1$ for $P \in \operatorname{Ass}_{A}(A / \pi A)$ " in the above result can be replaced by the condition " $A$ is $R$-flat".

Corollary 3.8. Let $(R, m)$ be a complete two-dimensional regular local domain with residue field $k$. Suppose that the algebraic closure $\bar{k}$ of $k$ is a finite extension of $k$. Let $A$ be a Noetherian domain such that $A$ is a flat $R$-algebra with $\operatorname{tr} \cdot \operatorname{deg}_{R} A=1$. Suppose that there exists $\pi \in m$ such that $A\left[\pi^{-1}\right]$ is a finitely generated $R\left[\pi^{-1}\right]$-algebra and tr.deg $\operatorname{deg}_{R /(P \cap R)} A / P>0$ for each $P \in \operatorname{Ass}_{A}(A / \pi A)$. Then $A$ is finitely generated over $R$.

Proof. Let $\pi(\neq 0) \in m, P \in \operatorname{Ass}_{A}(A / \pi A)$ and $p=P \cap R$. Since $A$ is $R$-flat, we have $\operatorname{depth} R_{p} \leq \operatorname{depth} A_{P}=1$. Hence $\operatorname{depth} R_{p}=1$, which implies that ht $p=1$, because $R$ is regular. The result now follows from Corollary 3.7.

Remark 3.9. The respective proofs show that the condition " $\operatorname{tr} \cdot \operatorname{deg}_{R /(P \cap R)} A / P>0$ for every $P \in \operatorname{Ass}_{A}(A / \pi A)$ " occurring in the above results may be replaced by the equivalent condition "tr. $\operatorname{deg}_{R /(P \cap R)} A / P=1$ for every $P \in \operatorname{Ass}_{A}(A / \pi A)$ ".

With the same notation and assumptions as in Theorem [3.6, we shall now give sufficient conditions for the ring $A$ to be Noetherian in the case where tr. $\operatorname{deg}_{R / p} A / P=0$ for every $P \in \operatorname{Ass}_{A}(A / \pi A)$ (Theorem 3.14). We begin by recording an auxiliary result.

Lemma 3.10. Let $(S, \mathfrak{n})$ be a complete one-dimensional Noetherian local domain whose residue field $k$ satisfies the condition that $[\bar{k}: k]<\infty$. Suppose that $B$ is a Noetherian domain such that $S \subseteq B$, tr. $\operatorname{deg}_{S} B=0$ and $\mathfrak{n} B \neq B$. Then $B$ is a finite $S$-module.

Proof. Since $B$ is a Noetherian domain, $B$ is separated for the $\mathfrak{n}$-adic topology. Thus, by $[9$, Theorem 8.4], it suffices to show that $B / \mathfrak{n} B$ is a finite $k$-module.

Let $\mathfrak{n} B=Q_{1} \cap Q_{2} \cap \cdots \cap Q_{n}$ be an irredundant primary decomposition of $\mathfrak{n} B$ in $B$ and let $P_{i}=\sqrt{Q_{i}}$ for $i=1, \ldots, n$. Then $P_{i} \cap S=\mathfrak{n}$ for each $i$, because $\operatorname{dim} S=1$. Since $\operatorname{tr} \cdot \operatorname{deg}_{S} B=0$, it then follows from the dimension inequality that

$$
\text { ht } P_{i}+\text { tr. } \operatorname{deg}_{k} B / P_{i} \leq \text { ht } \mathfrak{n}+\text { tr. } \operatorname{deg}_{S} B=1
$$


for each $i$. From this we have that ht $P_{i}=1$ and $\operatorname{tr} \cdot \operatorname{deg}_{k} B / P_{i}=0$, so that $B / P_{i}$ is a finite $k$-module for each $i$, because $[\bar{k}: k]<\infty$. Since $P_{i}^{r} \subseteq Q_{i}$ for some $r>0$, it thus follows that each $B / Q_{i}$ is a finite $k$-module. Note that

$$
B / \mathfrak{n} B \hookrightarrow B / Q_{1} \times B / Q_{2} \times \cdots \times B / Q_{n}
$$

is a finite extension. Therefore $B / \mathfrak{n} B$ is a finite $k$-module, as desired.

Lemma 3.11. Let $(S, \mathfrak{n})$ be a one-dimensional local domain and $B$ an integral domain containing $S$ such that $\mathfrak{n} B=B$ and $\operatorname{tr} \cdot \operatorname{deg}_{S} B=0$. Then $B$ is a field.

Proof. Let $P$ be an arbitrary prime ideal of $B$. Since $(0)$ and $\mathfrak{n}$ are the only prime ideals of the one-dimensional local domain $(S, \mathfrak{n})$ and $\mathfrak{n} B=B$, we have $P \cap S=(0)$.

Let $K$ denote the field of fractions of $S$. Since $P \cap S=(0)$, we have $K \subseteq B_{P}$. Since $\operatorname{tr} \cdot \operatorname{deg}_{S} B=0$, it then follows that $B_{P}$ is algebraic over the field $K$. Hence $B_{P}$ is a field, which implies that $P=(0)$. Thus, $B$ is a field.

Corollary 3.12. Let $(R, m)$ be a two-dimensional local domain and $A$ a Krull domain such that $R \subseteq A, m A=A$ and $\operatorname{tr} \cdot \operatorname{deg}_{R} A>0$. Let $\pi$ be a nonzero element in $m$ and $P$ a minimal prime ideal of $\pi A$. Suppose that tr. $\operatorname{deg}_{R /(P \cap R)} A / P=0$. Then $A / P$ is a field.

Proof. Set $p:=P \cap R, S:=R / p, \mathfrak{n}:=m / p$ and $B:=A / P$. Then $S \hookrightarrow B$ and identifying $S$ with its image in $B$, we may assume that $S \subseteq B$. Since $m A=A$, we have $\mathfrak{n} B=B$. The result now follows from Lemma 3.11 .

Proposition 3.13. Let $(R, m)$ be a two-dimensional complete Noetherian local domain whose residue field $k$ satisfies the condition that $[\bar{k}: k]<\infty$, and let $\pi$ be a nonzero prime element of $m$. Let $D$ be an integral domain containing $R$ such that $D / \pi D$ is a Noetherian domain, $\bigcap_{n \geq 0} \pi^{n} D=0$ and $\pi D \cap R=\pi R$. Then the following assertions hold.

(1) If tr. $\operatorname{deg}_{R / \pi R} D / \pi D=0$ and $m D \neq D$, then $D$ is a finite $R$-module.

(2) If tr. $\operatorname{deg}_{R} D>0$, then either tr. $\operatorname{deg}_{R / \pi R} D / \pi D>0$ or $m D=D$.

(3) If tr. $\operatorname{deg}_{R / \pi R} D / \pi D=0$ and $m D=D$, then $D / \pi D$ is a field. In addition, if $D\left[\pi^{-1}\right]$ is Noetherian, then $D$ is Noetherian.

Proof. Set $S=R / \pi R, \mathfrak{n}=m / \pi R$ and $B=D / \pi D$. Then, by assumptions, $(S, \mathfrak{n})$ is a complete one-dimensional Noetherian local domain with residue field $k$ satisfying the condition that $[\bar{k}: k]<\infty, B$ is a Noetherian domain and $S \hookrightarrow B$ so that, identifying $S$ with its image in $B$, we may assume that $S \subseteq B$.

(1) Note that $\mathfrak{n} B \neq B$ because $m D \neq D$. Since tr. $\operatorname{deg}_{S} B=0$, it follows from Lemma 3.10 that $B$ is a finite $S$-module. On the other hand, since $R$ is $m$-adically complete and $\pi \in m$, $R$ is $\pi$-adically complete, too. Since $\bigcap_{n \geq 0} \pi^{n} D=0$, it now follows from [9, Theorem 8.4] that $D$ is a finite $R$-module.

(2) The assertion follows from (1).

(3) The assertions follow from Corollary 3.12 and Lemma 2.4 respectively. 
We now state our result giving criteria for the $\operatorname{ring} A$ to be Noetherian when $A / P$ is algebraic over $R /(P \cap R)$ for every minimal prime $P$ of $\pi A$.

Theorem 3.14. Let $(R, m)$ be a two-dimensional complete Noetherian local domain whose residue field $k$ satisfies the condition that $[\bar{k}: k]<\infty$. Let $A$ be a Krull domain such that $R \subseteq A$ with $\operatorname{tr} \cdot \operatorname{deg}_{R} A>0$. Suppose that the following conditions hold.

(I) There exists $\pi \in m$ such that $A\left[\pi^{-1}\right]$ is Noetherian.

(II) For every minimal prime ideal $P$ of $\pi A, \operatorname{ht}(P \cap R)=1$ and $\operatorname{tr} \cdot \operatorname{deg}_{R /(P \cap R)} A / P=0$.

Then the following statements are equivalent.

(i) A is Noetherian.

(ii) $A / \pi A$ is Noetherian.

(iii) $m A=A$.

Proof. (i) $\Rightarrow$ (ii) is obvious.

(ii) $\Rightarrow$ (iii). Suppose on the contrary that $m A \neq A$, and let $M$ be a maximal ideal of $A$ such that $m A \subseteq M$. Set $A^{\prime}=A_{M}$. Then $A^{\prime}$ is a Krull local domain satisfying $m A^{\prime} \neq A^{\prime}$. Let $P^{\prime} \in \operatorname{Ass}_{A^{\prime}}\left(A^{\prime} / \pi A^{\prime}\right), P=P^{\prime} \cap A$ and $p=P \cap R$. Then $P \in \operatorname{Ass}_{A}(A / \pi A)$ and $A^{\prime} / P^{\prime}$ is a localisation of $A / P$, and hence tr. $\operatorname{deg}_{R / p} A^{\prime} / P^{\prime}=0$ and $A^{\prime} / P^{\prime}$ is Noetherian by our hypothesis. Note that $R / p$ is a complete one-dimensional local domain with residue field $k$ satisfying the condition that $[\bar{k}: k]<\infty$. Since $\operatorname{tr} \cdot \operatorname{deg}_{R / p}\left(A^{\prime} / P^{\prime}\right)=0$ and $m\left(A^{\prime} / P^{\prime}\right) \neq A^{\prime} / P^{\prime}$, it now follows from Lemma 3.10 that $A^{\prime} / P^{\prime}$ is a finite $R / p$-module. Therefore, by Lemma 3.5, we know that $A^{\prime} / \pi A^{\prime}$ is a finite $R$-module, which means $A^{\prime} / \pi A^{\prime}$ is a finite $R / \pi R$-module because $\pi R \subseteq \pi A^{\prime} \cap R$. Note that $\cap_{n \geq 0} \pi^{n} A^{\prime}=(0)$ because $A^{\prime}$ is a Krull domain. Note also that $R$ is complete with respect to $\pi$-adic topology because $\pi \in m$. It thus follows from [9, Theorem 8.4] that $A^{\prime}$ is a finite $R$-module, which contradicts the condition that $\operatorname{tr} \cdot \operatorname{deg}_{R} A^{\prime}>0$. Therefore $m A=A$.

(iii) $\Rightarrow$ (i). To prove that $A$ is Noetherian, it suffices to prove, by the Mori-Nishimura Theorem ([9, Theorem 12.7]) that $A / N$ is Noetherian for every prime ideal $N$ in $A$ of height one.

Fix a prime ideal $N$ in $A$ of height one. If $\pi \in N$, then $N$ is a minimal prime ideal of $\pi A$ and hence, $A / N$ is a field by Corollary 3.12 , in particular, $A / N$ is Noetherian.

Now we consider the case $\pi \notin N$. Since $A$ is a Krull domain and $P$ is a maximal ideal for every $P \in \operatorname{Ass}_{A}(A / \pi A)$ by Corollary 3.12 , it then follows that $N$ and $\pi A$ are comaximal ideals. Therefore, $A / N=A\left[\pi^{-1}\right] / N\left[\pi^{-1}\right]$, so that $A / N$ is Noetherian, since $A\left[\pi^{-1}\right]$ is Noetherian.

Thus, $A$ is Noetherian.

Remark 3.15. (1) The ring $A$ in Example 4.4 shows the necessity of the hypothesis that "tr. $\operatorname{deg}_{R / p R} A / P>0$ for each $P \in \operatorname{Ass}_{A}(A / \pi A)$ " in Theorem 3.6 .

(2) The ring $D$ in Example 4.5 shows the necessity of the hypothesis " $R$ is complete" in Proposition 3.13 .

(3) The hypothesis $[\bar{k}: k]<\infty$, occurring in most of the results in this section, is equivalent to the condition $[\bar{k}: k] \leq 2$; this condition is satisfied if and only if $k$ is either an algebraically closed field or a real closed field (cf. [4, Remark 4.3(2)]) 


\section{Examples}

In this section we shall give an example (Example 4.4) of a Noetherian normal non-finitely generated subalgebra of the polynomial ring $R[X]$ over a two-dimensional complete regular local ring $R$.

We shall first give methods (Lemmas 4.144.3) for constructing Noetherian normal $R$ subalgebras of $R[X]$, when $R$ is a Noetherian normal domain with field of fractions $K$. Lemma 4.1 considers a Krull subring $D$ of $K[X]$ with certain properties, Lemma 4.2 examines the ring $A:=D \cap R[X]$ and Lemma 4.3 gives a sufficient criterion for $D$ and $A$ to be Noetherian. These results are generalisations of Lemma 5.4 in [4].

Throughout this section we denote by $X$ an indeterminate over $R$.

Lemma 4.1. Let $R$ be a Noetherian normal domain, and let $\pi$ be a nonzero prime element of $R$. Let $D$ be an integral domain containing $R$ such that

(I) $D[1 / \pi]=R[1 / \pi][X]$;

(II) $\pi D$ is a prime ideal and $\pi D \cap R=\pi R$;

(III) $D_{(\pi D)}$ is a discrete valuation ring.

Then the following statements hold.

(1) D is a Krull domain.

(2) If $R$ a UFD then $D$ is a UFD.

(3) If $p$ is a prime element of $R$, then $p$ remains a prime element in $D$ and either $p R=\pi R$ or $p D_{(\pi D)}=D_{(\pi D)}$.

Proof. (1) By Lemma 2.3, $D=D[1 / \pi] \cap D_{(\pi D)}$. Hence $D$ is Krull, because both $D[1 / \pi]$ and $D_{(\pi D)}$ are Noetherian normal domains by (I) and (III).

(2) Now suppose that $R$ is a UFD. Then $D[1 / \pi]$ is a UFD by (I). Since $D$ is a Krull domain, $\pi$ is a prime element of $D$ and $D[1 / \pi]$ is a UFD, it follows that $D$ is a UFD by Nagata's criterion ([6, Corollary 7.3]).

(3) Let $p$ be a nonzero prime element of $R$. If $p R=\pi R$, then $p D=\pi D$, so that $p$ is prime in $D$ by (II).

So we consider the case $p R \neq \pi R$. In this case, $p \notin \pi R$, and hence $p \notin \pi D$ because of (II), which implies that $p D_{(\pi D)}=D_{(\pi D)}$.

Since $D=D[1 / \pi] \cap D_{(\pi D)}$, it thus follows that $p D=p D[1 / \pi] \cap D$. Note that $p$ is a prime element of $R[1 / \pi]$ because $p R \neq \pi R$, and hence $p$ is prime in $R[1 / \pi][X]=D[1 / \pi]$. Thus $p D[1 / \pi]$ is a prime ideal of $D[1 / \pi]$, which implies that $p D(=p D[1 / \pi] \cap D)$ is a prime ideal of $D$, as desired. This completes the proof.

Lemma 4.2. Let $R, \pi$ and $D$ be as in Lemma 4.1 (with conditions (I), (II) and (III)). Suppose that $R[X] \nsubseteq D$ and $D \nsubseteq R[X]$. Set $A:=R[X] \cap D, P_{1}:=\pi R[X] \cap A$ and $P_{2}:=\pi D \cap A$. Then the following assertions hold. 
(1) $A$ is a Krull domain. Further, $A=R[X] \cap D_{(\pi D)}$ and $A[1 / \pi]=R[1 / \pi][X](=D[1 / \pi])$.

(2) $\pi A=P_{1} \cap P_{2}, P_{1} \nsubseteq P_{2}$, and $P_{2} \nsubseteq P_{1}$.

(3) $A_{P_{1}}=R[X]_{(\pi R[X])}$ and $A_{P_{2}}=D_{(\pi D)}$.

(4) For each $f \in P_{1}, A[1 / f]=D[1 / f]$ and for each $f \in P_{2}, A[1 / f]=R[X][1 / f]$.

(5) For any prime element $p$ in $R$ with $p R \neq \pi R, p A=p R[X] \cap A$ and hence $p$ remains a prime element in $A$.

(6) If $(R, m)$ is a two-dimensional regular local ring with $m=(\pi, t) R$, then $A$ is a faithfully flat $R$-algebra.

Proof. (1) Since $D$ is a Krull domain by Lemma 4.1(1) and $R$ is a Noetherian normal domain, $A(=R[X] \cap D)$ is a Krull domain.

Since $D[1 / \pi]=R[1 / \pi][X]$, we have $A[1 / \pi]=R[1 / \pi][X] \cap D[1 / \pi]=R[1 / \pi][X]$. Moreover, since $D=D[1 / \pi] \cap D_{(\pi D)}$ by Lemma 2.3 and $R[X] \subseteq D[1 / \pi]$, we have

$$
A=R[X] \cap D=R[X] \cap\left(D[1 / \pi] \cap D_{(\pi D)}\right)=R[X] \cap D_{(\pi D)} .
$$

(2) Since $\pi A=\pi R[X] \cap \pi D$, we have $\pi A=P_{1} \cap P_{2}$.

We shall show that $P_{1} \nsubseteq P_{2}$ by contradiction. Suppose, if possible, that $P_{1} \subseteq P_{2}$. Then $\pi A=P_{1}=\pi R[X] \cap A$, while $A[1 / \pi]=R[X][1 / \pi]$ by (1). Since $A \subseteq R[X]$, it thus follows from Lemma 2.3 that $A=R[X]$. This implies that $R[X] \subseteq D$, which contradicts our hypothesis.

Similarly we have $P_{2} \nsubseteq P_{1}$.

(3) Since $A$ is a Krull domain by (1) and $\pi A=P_{1} \cap P_{2}$ is the irredundant prime decomposition of $\pi A$ in $A$ by (2), it follows that $A_{P_{i}}$ is a DVR for $i=1,2$. Therefore $A_{P_{1}}=R[X]_{(\pi R[X])}$ and $A_{P_{2}}=D_{(\pi D)}$, because $A_{P_{1}} \subseteq R[X]_{(\pi R[X])}, A_{P_{2}} \subseteq D_{(\pi D)}$ and $A[1 / \pi]=R[1 / \pi][X]=D[1 / \pi]$.

(4) Let $f \in P_{2}$. Clearly $A[1 / f] \subseteq R[X][1 / f]$ and by (2), $\pi A[1 / f]=P_{1} A[1 / f]=$ $\pi R[X][1 / f] \cap A[1 / f]$. Since $A[1 / f][1 / \pi]=R[X][1 / f, 1 / \pi]$ by (1), it follows from Lemma 2.3 that $A[1 / f]=R[X][1 / f]$. Similarly, we have $A[1 / f]=D[1 / f]$ for each $f \in P_{1}$.

(5) Since $p R \neq \pi R$, we have $p D_{(\pi D)}=D_{(\pi D)}$ by Lemma 4.1(3). It then follows from (1) that $p A=p R[X] \cap D_{(\pi D)}=p R[X] \cap A$. Thus $p$ is prime in $A$.

(6) By (5), $t$ is a prime element in $A$ and hence $\{t, \pi\}$ is a regular sequence in $A$. Therefore, by Lemma 2.9, $A$ is a flat $R$-algebra. Since $A \subseteq R[X]$, it follows that $A$ is faithfully flat over $R$.

Lemma 4.3. Let $R, D, A, P_{1}$ and $P_{2}$ be as in Lemmas 4.1 and 4.2. Then the following statements hold.

(1) If $P_{1}+P_{2}=A$, then $A / \pi A \cong A / P_{1} \times A / P_{2}, A / P_{1} \cong(R / \pi R)[X]$ and $A / P_{2} \cong D / \pi D$.

(2) If $\pi D$ is a maximal ideal of $D$, then $D$ is a Noetherian domain.

(3) If $D$ is a Noetherian domain, then $A[1 / f]$ is Noetherian for every $f \in P_{1}+P_{2}$. In particular, if $P_{1}+P_{2}=A$, then $A$ is a Noetherian domain. 
(4) If $D$ is a finitely generated $R$-algebra, then $A[1 / f]$ is a finitely generated $R$-algebra for every $f \in P_{1}+P_{2}$. In particular, if $P_{1}+P_{2}=A$ then $A$ is a finitely generated $R$-algebra.

Proof. (1) Assume $P_{1}+P_{2}=A$. Then, since $\pi A=P_{1} \cap P_{2}$ by Lemma 4.2(2), we have $A / \pi A \cong A / P_{1} \times A / P_{2}$.

We now show that $A / P_{1} \cong R[X] / \pi R[X]$, namely, $R[X]=A+\pi R[X]$. Let $f \in R[X]$. Since $P_{1}+P_{2}=A$, there exist $a \in P_{1}$ and $b \in P_{2}$ such that $a+b=1$. Then $A\left[b^{-1}\right]=R[X]\left[b^{-1}\right]$ by Lemma 4.2(4), so that $b^{n} f=(1-a)^{n} f \in A$ for some $n>0$. Since $a \in P_{1} \subseteq \pi R[X]$, from this it follows that $f \in A+\pi R[X]$, as desired.

Similarly we have $A / P_{2} \cong D / \pi D$.

(2) Since $D[1 / \pi](=R[1 / \pi][X])$ is Noetherian and $\operatorname{ht}(\pi D)=1$ (as $D_{(\pi D)}$ is a DVR), the assertion follows from Lemma 2.4 .

(3) Let $f \in P_{1}+P_{2}$, and write $f=a+b$ with $a \in P_{1}$ and $b \in P_{2}$. Then $A[1 / a]=D[1 / a]$ and $A[1 / b]=R[X][1 / b]$ by Lemma $4.2(4)$, so that both $A[1 / a]$ and $A[1 / b]$ are Noetherian. Since $(a, b) A[1 / f]=A[1 / f]$, it then follows from Lemma $2.5(1)$ that $A[1 / f]$ is Noetherian.

(4) The proof is similar to the above proof of (3).

We now present our main example over the complete regular local domain $R=\mathbb{C}[[u, v]]$, where $u$ and $v$ are indeterminates over $\mathbb{C}$ : we construct a Noetherian normal $R$-subalgebra of $R[X]$ which is not finitely generated over $R$. This example shows that Theorem 1.1 does not extend to complete local rings of dimension two and that the hypothesis on the transcendence degree of certain fibres is necessary in Theorem 3.6 .

Example 4.4. Let $R=\mathbb{C}[[u, v]]$, where $u$ and $v$ are indeterminates over $\mathbb{C}$, and let $p_{n}$ denote the $n$-th prime number for $n>0$. We set

$$
L:=\mathbb{C}((v))\left[v^{1 / p_{1}}, v^{1 / p_{2}}, \ldots, v^{1 / p_{n}}, \ldots\right],
$$

so that $L$ is an infinite algebraic extension of the field $\mathbb{C}((v))$.

Let $x_{0}=u X, x_{1}=\left(v x_{0}-1\right) / u$, and

$$
x_{n}=\frac{x_{n-1}^{p_{n-1}}-v}{u}
$$

for $n>1$. Let $D=R\left[x_{0}, x_{1}, \ldots, x_{n}, \ldots\right]$ and $A=R[X] \cap D$. Then the following hold.

(1) $D[1 / u]=R[1 / u][X]$.

(2) $D / u D \cong L$; in particular, $D / u D$ is not finitely generated over $R / u R$.

(3) $u D$ is a maximal ideal of $D, u D \cap R=u R$ and $\operatorname{ht}(u D)=1$.

(4) $D$ is a Noetherian UFD which is not finitely generated over $R$.

(5) A is a Noetherian normal domain which is faithfully flat but not finitely generated over $R$. 
Proof. (1) Since $x_{0}=u X$ and $x_{i} \in R[1 / u][X]$ for each $i>0$, we have $D[1 / u]=R[1 / u][X]$.

(2) Set

$$
f_{1}:=u X_{1}-v X_{0}+1 \text { and } f_{n}:=u X_{n}-X_{n-1}^{p_{n-1}}+v \text { for } n \geq 2,
$$

where $X_{0}, X_{1}, \ldots, X_{n}, \ldots$ are indeterminates over $R$. Let

$$
I_{n}=\left(f_{1}, f_{2}, \ldots, f_{n}\right) R\left[X_{0}, X_{1}, \ldots, X_{n}\right] \text { for } n \geq 1
$$

and let $I_{0}$ denote the zero ideal of $R\left[X_{0}\right]$. Set

$$
C_{0}:=R\left[X_{0}\right] \text { and } C_{n}:=R\left[X_{0}, X_{1}, \cdots, X_{n}\right] / I_{n} \text { for } n \geq 1
$$

We shall prove, by induction on $n$, that for each $n \geq 1, I_{n} \cap R\left[X_{0}, X_{1}, \ldots, X_{n-1}\right]=I_{n-1}$ and $I_{n}$ is a prime ideal of $R\left[X_{0}, X_{1}, \ldots, X_{n}\right]$ with $I_{n} \cap R=(0)$. This would establish that each $C_{n}$ is an integral domain and that we may identify $C_{n-1}$ with its canonical image in $C_{n}$, i.e., we may assume that, for every $n \geq 1$,

$$
R \subseteq C_{0} \subseteq \cdots \subseteq C_{n-1} \subseteq C_{n}
$$

Note that $f_{1}$ is an irreducible polynomial in $R\left[X_{0}, X_{1}\right]$ which is a UFD so that $I_{1}$ is a prime ideal of $R\left[X_{0}, X_{1}\right]$. Further $I_{1} \cap C_{0}=(0)$ and $I_{1} \cap R=(0)$. Thus the assertion holds for $n=1$.

Now suppose that the assertion holds for $n$. Let $J=I_{n+1} \cap R\left[X_{0}, \ldots, X_{n}\right]$. We first show that $J=I_{n}$. Clearly, $I_{n} \subseteq J$. Note that

$$
R\left[u^{-1}\right]\left[X_{0}, X_{1}, \ldots, X_{m}\right]=R\left[u^{-1}\right]\left[X_{0}, f_{1}, \ldots, f_{m}\right]
$$

for every $m$, which implies that $J\left[u^{-1}\right]=I_{n}\left[u^{-1}\right]$. Now let $h \in J$. Then $u^{r} h \in I_{n}$ for some $r>0$. By induction hypothesis, $I_{n}$ is a prime ideal of $R\left[X_{0}, X_{1}, \cdots, X_{n}\right]$ with $I_{n} \cap R=(0)$. In particular, $u \notin I_{n}$ as $u \in R$, so that $h \in I_{n}$. Thus we have $J=I_{n}$, as claimed. Therefore, we may assume that $C_{n} \subseteq C_{n+1}$. Note that, $I_{n+1} \cap R=I_{n+1} \cap R\left[X_{0}, \ldots, X_{n}\right] \cap R=I_{n} \cap R=(0)$.

We next show that $I_{n+1}$ is a prime ideal of $R\left[X_{0}, X_{1}, \cdots, X_{n+1}\right]$, i.e., $C_{n+1}$ is an integral domain. Let $z_{n}$ denote the image of $X_{n}$ in $C_{n}$, set $w_{n}:=z_{n}{ }^{p_{n}}-v \in C_{n}$ and

$$
g_{n+1}:=u X_{n+1}-w_{n} \in C_{n}\left[X_{n+1}\right]
$$

i.e., $g_{n+1}$ is the image of $f_{n+1}$ in $C_{n}\left[X_{n+1}\right]$. Since $C_{n} \subseteq C_{n+1}$, it then follows that

$$
C_{n+1}=C_{n}\left[z_{n+1}\right] \cong C_{n}\left[X_{n+1}\right] /\left(g_{n+1}\right) \text { and } C_{n+1}[1 / u] \cong C_{n}[1 / u] .
$$

Also setting $L_{n}:=\mathbb{C}((v))\left[v^{1 / 2}, v^{1 / 3}, \ldots, v^{1 / p_{n}}\right]$, an algebraic extension of $\mathbb{C}((v))$, we have

$$
C_{n} / u C_{n} \cong \mathbb{C}[[v]]\left[X_{0}, X_{1}, \ldots, X_{n}\right] /\left(v X_{0}-1, X_{1}^{2}-v, \ldots, X_{n-1}^{p_{n-1}}-v\right) \cong L_{n-1}\left[X_{n}\right]
$$

and hence $C_{n} / u C_{n}$ is an integral domain. This implies that $u, w_{n}$ is a regular sequence in $C_{n}$ and hence a regular sequence in $C_{n}\left[X_{n+1}\right]$. Thus, $u$ is a regular element of $C_{n+1}$, so that 
the canonical map $C_{n+1} \rightarrow C_{n+1}[1 / u]$ is injective. But $C_{n+1}[1 / u]\left(\cong C_{n}[1 / u]\right)$ is an integral domain. Therefore, $C_{n+1}$ is an integral domain, as desired.

Since $w_{n}$ is the image of $X_{n}{ }^{p_{n}}-v$ in $C_{n}$, we have

$$
C_{n} /\left(u, w_{n}\right) C_{n} \cong \mathbb{C}[[v]]\left[X_{0}, X_{1}, \ldots, X_{n}\right] /\left(v X_{0}-1, X_{1}^{2}-v, \ldots, X_{n-1}{ }^{p_{n-1}}-v, X_{n}{ }^{p_{n}}-v\right) \cong L_{n} .
$$

Thus, we have a canonical isomorphism

$$
\psi_{n}: C_{n} /\left(u, w_{n}\right) C_{n} \stackrel{\sim}{\rightarrow} L_{n} .
$$

and hence a canonical surjection $\theta_{n}: C_{n} \rightarrow L_{n}$.

Now, let $C=\bigcup_{n \geq 0} C_{n}$. As $C_{0}=R^{[1]}$ and $C_{n+1}$ is algebraic over $C_{n} \forall n \geq 0$, we have tr. $\operatorname{deg}_{R} C=1$. For each $n \geq 0$, let

$$
\Phi_{n}: R\left[X_{0}, X_{1}, \ldots, X_{n}\right] \rightarrow D
$$

be the $R$-algebra map defined by

$$
\Phi_{n}\left(X_{i}\right)=x_{i} \text { for } 0 \leq i \leq n .
$$

Then, for each $j, 1 \leq j \leq n, \Phi_{n}\left(f_{j}\right)=u x_{j}-x_{j-1}{ }^{p_{j-1}}+v=0$, so that $\Phi_{n}$ induces an $R$-algebra map

$$
\phi_{n}: C_{n} \rightarrow D
$$

such that

$$
\phi_{n}\left(z_{i}\right)=x_{i} \text { for } 0 \leq i \leq n .
$$

In particular, for any $m \geq 0$, we have

$$
\phi_{m}\left(z_{m}\right)=x_{m}
$$

Since tr. $\operatorname{deg}_{R} C_{n}=1=\operatorname{tr} \cdot \operatorname{deg}_{R} D$, and since both $C_{n}$ and $D$ are integral domains, it then follows that $\phi_{n}$ is an injective $R$-algebra homomorphism. We thus have an injective $R$-algebra map

$$
\phi: C \rightarrow D \text { such that }\left.\phi\right|_{C_{n}}=\phi_{n}
$$

which is also surjective as, for each $m \geq 0, \phi\left(z_{m}\right)=\phi_{m}\left(z_{m}\right)=x_{m}$ by (4.2). Thus, $C \cong D$.

Note that

$$
\phi_{n}\left(w_{n}\right)=x_{n}^{p_{n}}-v=u x_{n+1} \in u D,
$$

so that $\phi_{n}\left(u, w_{n}\right) C_{n} \subseteq u D$. Hence, as $L_{n}$ is a field, by the isomorphism $\psi_{n}$ in (4.1), $\phi_{n}$ induces an injective map

Note that, for any $m \geq 0$,

$$
\bar{\phi}_{n}: L_{n} \rightarrow \frac{D}{u D}
$$

$$
\overline{\phi_{m}}\left(\theta_{m}\left(z_{m}\right)\right)=\overline{x_{m}}
$$

where $\overline{x_{m}}$ denotes the image of $x_{m}$ in $D / u D$. Now, the $\overline{\phi_{n}}$ 's give rise to an injective $R$-algebra map

$$
\bar{\phi}: L \rightarrow \frac{D}{u D}
$$


which is also surjective as, given any $m \geq 0, \bar{\phi}\left(\theta_{m}\left(z_{m}\right)\right)=\overline{x_{m}}$ by (4.3). Thus $\bar{\phi}$ is an isomorphism, i.e., $D / u D \cong L$.

As $L(\cong D / u D)$ is algebraic over $\mathbb{C}[[v]](\cong R / u R)$ and hence over $\mathbb{C}((v))$, and as $L$ is not a finite extension of $\mathbb{C}((v))$, it follows that $D / u D$ is not a finitely generated algebra over $R / u R$.

(3) $u D$ is a maximal ideal by (2).

Since $\mathbb{C}[[v]] \subseteq L$, the canonical map from $R / u R(=\mathbb{C}[[v]])$ to $D / u D(=L$ by $(2))$ is injective. Hence $u D \cap R=u R$.

We show that $\operatorname{ht}(u D)=1$. Since $R$ is a Noetherian domain and $u D \cap R=u R$, it follows from Theorem 2.1 that

$$
\operatorname{ht}(u D)+\operatorname{tr} \cdot \operatorname{deg}_{R / u R}(D / u D) \leq \operatorname{ht}(u R)+\operatorname{tr} \cdot \operatorname{deg}_{R} D=2,
$$

so that $\operatorname{ht}(u D) \leq 2$. Now tr. $\operatorname{deg}_{R / u R}(D / u D)=0$ by $(2)$. Therefore, if ht $(u D)=2$, then $u D$ would satisfy the dimension formula relative to $R$ and hence, by [10, Theorem 3.6], it would follow that $D / u D$ is a subalgebra of a finitely generated $(R / u R)$-algebra. But then, as $D / u D$ is a field by (2), it would follow from Proposition 2.7 that $D / u D$ itself is a finitely generated $R / u R$-algebra, which is not the case by $(2)$. Hence, ht $(u D)=1$.

(4) From (1) and (3), it follows, by Lemma 2.4, that $D$ is Noetherian. $D$ is a UFD by Lemma 4.1(2). Since $D / u D$ is not finitely generated over $R / u R$ by (2), it follows that $D$ is not finitely generated over $R$.

(5) $A$ is faithfully flat over $R$ by Lemma $4.2(6)$.

$A$ is a Krull domain by Lemma 4.2(1), so that $A$ is normal.

We show that $A$ is Noetherian. Let $P_{1}=u R[X] \cap A$ and $P_{2}=u D \cap A$ (as in Lemma 4.2). Since $x_{0}=u X \in u R[X] \cap A=P_{1}$ and $v x_{0}-1\left(=u x_{1}\right) \in u D \cap A=P_{2}$, we have $P_{1}+P_{2}=A$. Thus $A$ is Noetherian by Lemma 4.3(3).

Since $D / u D$ is not finitely generated over $R / u R$ by (2) and $A / P_{2} \cong D / u D$ by Lemma 4.3 (1), it follows that $A / P_{2}$ is not finitely generated over $R / u R$ and hence $A$ is not finitely generated over $R$.

The following example shows that the condition in Lemma 4.3 that $D / \pi D$ is a field cannot be replaced by the condition that $D / \pi D$ is Noetherian in order to conclude that $D$ is Noetherian. It also illustrates the necessity of the hypothesis that $R$ is complete in Proposition 3.13 ,

Example 4.5. Let $R=k[u, v]_{(u, v)}$, where $k=\overline{\mathbb{Q}}$ is the algebraic closure of $\mathbb{Q}$ and $u, v$ are indeterminates over $k$. Set $a_{0}:=1$ and $a_{n}:=v^{n} / n$ ! for $n>0$. Set $x_{0}:=u X$, where $X$ is an indeterminate over $R$ and

$$
x_{n}:=\frac{x_{n-1}-a_{n-1}}{u}=\frac{x_{0}-a_{0}-a_{1} u-\cdots-a_{n-1} u^{n-1}}{u^{n}}
$$

for $n \geq 1$. Let $D=R\left[x_{0}, x_{1}, \ldots, x_{n}, \ldots\right]$. Then the following statements hold.

(1) $D[1 / u]=R[1 / u][X]$. 
(2) $u$ is a prime element in $D$ and $u D \cap R=u R$.

(3) $D / u D=R / u R$. In particular, $D / u D$ is Noetherian and $D /(u, v) D$ is a domain.

(4) $D_{(u D)}$ is a discrete valuation ring.

(5) $D$ is a non-Noetherian UFD.

Proof. We first note that $D=\bigcup_{n \geq 0} R\left[x_{n}\right]$ and for each $n \geq 0, x_{n}=u x_{n+1}+a_{n}$ for some $a_{n} \in R$. The assertions (1)-(3) follow from this.

(4) Set $\widetilde{R}:=k[v][[u]]_{(u, v)}$. Then $\widetilde{R}$ is a Noetherian ring being the $u$-adic completion of $R$. We show that $D$ is $R$-isomorphic to a subring $D^{\prime}$ of $\widetilde{R}$. We set

$$
y:=a_{0}+a_{1} u+a_{2} u^{2}+\cdots+a_{n} u^{n}+\cdots,
$$

so that $y$ is an element of $k[v][[u]]_{(u, v)}(=\widetilde{R})$. Since $a_{0}=1$ and $a_{n}=v^{n} / n$ ! for each $n>0$, it follows that

$$
y=1+\frac{v u}{1}+\frac{(v u)^{2}}{2 !}+\cdots+\frac{(v u)^{n}}{n !}+\cdots .
$$

Thus $y$ is a transcendental element over $R$. We now define a subring $D^{\prime}$ of $\widetilde{R}$ by

$$
D^{\prime}:=R\left[y, \frac{y-a_{0}}{u}, \frac{y-a_{0}-a_{1} u}{u^{2}}, \ldots, \frac{y-a_{0}-a_{1} u-\cdots-a_{n-1} u^{n-1}}{u^{n}}, \ldots\right] .
$$

Let $\phi: D \rightarrow D^{\prime}$ be the $R$-linear map defined by $\phi\left(x_{0}\right)=y$. Since $y$ is transcendental over $R$, it follows that $\phi$ is an isomorphism.

Note that we have $\bigcap_{n>0} u^{n} D^{\prime} \subseteq \bigcap_{n>0} u^{n} \widetilde{R}=(0)$. Since $\phi$ is an isomorphism, from this it follows that $\bigcap_{n>0} u^{n} D=0$, which implies that ht $(u D)=1$. As $D$ is a Krull domain by Lemma 4.1(1), it follows that $D_{(u D)}$ is a DVR.

(5) $D$ is a UFD by Lemma 4.1 (2). Note that $v \in R$ remains a prime element in $D$ by Lemma 4.1)(3). Since

$$
x_{0}-1=x_{0}-a_{0}=u^{n} x_{n}+a_{1} u+\cdots+a_{n-1} u^{n-1}
$$

and $a_{i} \in v R \subseteq v D$ for $i>0$, we have $x_{0}-1 \in \bigcap_{n>0} u^{n}(D / v D)$, which shows that the integral domain $D / v D$ is not Noetherian. Thus $D$ is not Noetherian.

Let $R$ be a Noetherian normal domain and $A$ be a Krull domain such that $R \subseteq A \subseteq R[X]$. If $\operatorname{dim} R=1$, then $A$ is a Noetherian domain by [4, Lemma 3.3]. The following example shows that the result does not hold when $\operatorname{dim} R=2$, not even if $R$ is a complete regular local ring.

Example 4.6. Let $R=\mathbb{C}[[u, v]]$, where $u$ and $v$ are indeterminates over $\mathbb{C}$. Let $p_{n}$ denote the $n^{\text {th }}$ prime number and set $q_{n}=\prod_{i=1}^{n} p_{i}$ for $n>0$. Let $S$ be the infinite integral extension of $\mathbb{C}[[v]]$ generated by all the $v^{1 / q_{n}}$ 's, i.e.,

$$
S=\bigcup_{n \geq 1} \mathbb{C}\left[\left[v^{1 / q_{n}}\right]\right]
$$


Set $x_{0}:=u X, x_{1}:=\left(x_{0}^{2}-v\right) / u$ and

$$
x_{n}=\frac{x_{n-1}^{p_{n}}-x_{n-2}}{u}
$$

for $n \geq 2$. Let $D=R\left[x_{0}, x_{1}, \ldots, x_{n}, \ldots\right]$ and $A=D \cap R[X]$. Then the following hold.

(1) $D[1 / u]=R[1 / u][X]$.

(2) $u D$ is a prime ideal of $D$ and $\operatorname{ht}(u D)=1$.

(3) $D / u D \cong S$, and hence $D / u D$ is a non-Noetherian ring.

(4) $D$ is a non-Noetherian UFD.

(5) A is a non-Noetherian Krull subalgebra of $R[X]$.

Proof. Proof of (1) follows easily. Following the same argument as in Example 4.4, one can show that $D / u D \cong C[[v]]\left[X_{0}, X_{1}, X_{2}, \ldots\right] /\left(X_{0}{ }^{2}-v, X_{1}{ }^{3}-X_{0}, X_{2}{ }^{5}-X_{1}, \ldots\right) \cong S$ and $\operatorname{ht}(u D)=1$. Note that $S=\bigcup_{n \geq 1} \mathbb{C}\left[\left[v^{1 / q_{n}}\right]\right]$ is a direct limit of discrete valuation rings $\mathbb{C}\left[\left[v^{1 / q_{n}}\right]\right]$ and hence a valuation ring. It is an infinite integral extension over $R / u R=\mathbb{C}[[v]]$. Further $S$ has following infinite ascending chain of ideals

$$
(v) \varsubsetneqq\left(v^{1 / 2}\right) \varsubsetneqq \cdots \varsubsetneqq\left(v^{1 / q_{n}}\right) \varsubsetneqq \cdots
$$

showing that $D / u D$ is not Noetherian. Thus (2) and (3) follow from above.

(4) $D$ is a UFD by Lemma 4.1. Since $D / u D$ is not Noetherian, $D$ is not Noetherian.

(5) $A$ is Krull domain by Lemma 4.2 (1). Note that $D$ and $A$ are of the "types" as in Lemmas 4.1 and 4.2, Recall that, $P_{1}=u R[X] \cap A$ and $P_{2}=u D \cap A$. Now $x_{0}=u X \in$ $u R[X] \cap A=P_{1}$ and $x_{0}{ }^{2}-v \in u D \cap A=P_{2}$ and so $v \in P_{1}+P_{2}$. Thus, by Lemma 4.3 (1), $\left(A / P_{2}\right)[1 / v]=(D / u D)[1 / v]$. Therefore, $A / P_{2}$ and $D / u D$ are birational. Therefore, since $A / P_{2}$ is a one dimensional domain and $D / u D$ is not a Noetherian ring, $A / P_{2}$ is not Noetherian by Krull-Akizuki theorem (cf. [9, Theorem 11.7]). Thus, $A$ is not a Noetherian ring.

\section{Appendix}

The following result on finite generation of algebras has been proved for the case $R$ is a field in [11, Theorem 1.1]. Below, we show that the result can be extended to an excellent domain. The proof is essentially the same as in [11].

Proposition 5.1. Let $R$ be an excellent local domain and $A$ a normal domain containing $R$. Suppose that there exists a nonzero element $f$ in A satisfying the following hypotheses.

(I) $A\left[f^{-1}\right]$ is a finitely generated $R$-algebra.

(II) $A / f A$ is a finitely generated $R$-algebra. 
(III) For every minimal prime ideal $P$ of $f$ in $A$, ht $P=1$ and $P$ satisfies the dimension formula relative to $R$.

Then $A$ is finitely generated over $R$.

Proof. Since $A\left[f^{-1}\right]$ is a finitely generated $R$-algebra, by Theorem 2.8(1), it is enough to show that $A_{M}$ is a locality (essentially of finite type) over $R$ for every maximal $M$ of $A$. Let $M$ be a maximal ideal of $A$. Since $A\left[f^{-1}\right]$ is finitely generated over $R$, we may assume that $f \in M$ for our consideration.

Let $P$ be a minimal prime ideal of $f$ in $A$ and $p=P \cap R$. Since $P$ satisfies the dimension formula relative to $R$, we have

$$
\text { ht } P+\text { tr. } \operatorname{deg}_{R / p} A / P=\text { ht } p+\text { tr. } \operatorname{deg}_{R} A .
$$

Since $R$ is an excellent ring, it is universally catenary. Hence, since, by (II), $A / P$ is a finitely generated $(R / p)$-algebra, we have

$$
\operatorname{ht}(M / P)+\operatorname{tr} \cdot \operatorname{deg}_{R /(M \cap R)} A / M=\operatorname{ht}((M \cap R) / p)+\operatorname{tr} \cdot \operatorname{deg}_{R / p} A / P
$$

by [9, Theorem 15.6]. From (5.1) and (5.2), and the fact that $R$ is catenary, we have

$$
\text { ht } P+\operatorname{ht}(M / P)=\operatorname{ht}(M \cap R)+\operatorname{tr} \cdot \operatorname{deg}_{R} A-\operatorname{tr} \cdot \operatorname{deg}_{R /(M \cap R)} A / M .
$$

By Theorem 2.1, we have

$$
\text { ht } M \leq \operatorname{ht}(M \cap R)+\text { tr. } \operatorname{deg}_{R} A-\operatorname{tr} \cdot \operatorname{deg}_{R /(M \cap R)} A / M .
$$

Hence by (5.3), we have

$$
\text { ht } P+\operatorname{ht}(M / P) \geq \text { ht } M \text {. }
$$

Thus, for any minimal prime ideal $P$ of $f$ in $A$,

$$
\text { ht } M=\operatorname{ht} P+\operatorname{ht}(M / P)=\operatorname{ht}(M / P)+1 \text {, }
$$

since ht $P=1$.

Let $\widehat{A}$ denote the $M$-adic completion of $A_{M}$. We show that $\widehat{A}$ is a Noetherian ring such that $\operatorname{dim} \widehat{A} \geq$ ht $M$. Let $\widetilde{A}$ be the $f$-adic completion of $A_{M}$. Then $\widetilde{A} / f \widetilde{A} \cong A_{M} / f A_{M}$, which is Noetherian by hypothesis (II). Hence $\widetilde{A}$ is Noetherian (cf. [14, Corollary 4, p.260]). Since $\widehat{A}$ coincides with the $M$-adic completion of $\widetilde{A}$, we have $\widehat{A}$ is a Noetherian ring and

$$
\operatorname{dim} \widehat{A}=\operatorname{dim} \widetilde{A} \text {. }
$$

On the other hand $f$ is a regular element of $\widetilde{A}$. Hence, by (5.4),

$$
\operatorname{dim} \widehat{A}=\operatorname{dim} \widetilde{A} \geq \operatorname{dim} \widetilde{A} / f \widetilde{A}+1=\operatorname{dim} A_{M} / f A_{M}+1=\operatorname{ht} M .
$$

Since $A\left[f^{-1}\right]$ and $A / f A$ are finitely generated $R$-algebras, there exists a finitely generated $R$-algebra $C$ such that $C\left[f^{-1}\right]=A\left[f^{-1}\right]$ and $C /(f A \cap C)=A / f A$. Since $f \in M, M$ is a finitely generated ideal of $A$ by hypothesis (II) and we may assume that the generators of $M$ are contained in $C$, so that $M=(M \cap C) A$. Let $B$ be the integral closure of $C$ in its field of fractions. Since $R$ is excellent and $C$ is a finitely generated $R$-algebra, $B$ is also finitely generated over $R$. Since $A$ is normal and birational to $C, B \hookrightarrow A$ and we have 
(1) $B\left[f^{-1}\right]=A\left[f^{-1}\right]$.

(2) $B /(f A \cap B)=A / f A$.

(3) $B / m=A / M$ and $m A=M$, where $m=M \cap B$.

(4) $B$ is a finitely generated normal $R$-algebra.

Let $\widehat{B}$ be the m-adic completion of $B_{m}$. Since $R$ is an excellent domain and $B$ is a finitely generated normal $R$-algebra, we have $\widehat{B}$ is a Noetherian normal domain ([8, Theorem 79, p. 258]). Let $\phi: \widehat{B} \rightarrow \widehat{A}$ be the canonical map induced by the inclusion $B_{m} \hookrightarrow A_{M}$. We show that $\phi$ is an isomorphism.

Since $\widehat{B} / m \widehat{B}=B / m=A / M=\widehat{A} / M \widehat{A}$ and $\widehat{A}$ is complete, we have $\phi$ is surjective (cf. [14, Corollary 2, p.259]). Now, since $\widehat{B}$ is an integral domain, to show that $\phi$ is injective, it is enough to show that $\operatorname{dim} \widehat{B} \leq \operatorname{dim} \widehat{A}$.

Since $B$ is a finitely generated $R$-algebra, we have

$$
\operatorname{dim} \widehat{B}=\operatorname{ht} m=\operatorname{ht}(m / Q)+\operatorname{ht} Q,
$$

where $Q=P \cap B$ and $P$ is a minimal prime ideal of $f$ in $A$. By (2) and (3), we have

$$
\operatorname{ht}(m / Q)=\operatorname{ht}(M / P) \text {. }
$$

Using (5.1), and the facts that $Q=P \cap B, B$ is birational to $A$ such that $B / Q=A / P$ and $Q$ satisfies the dimension formula relative to $R$, we have

$$
\text { ht } Q=\text { ht } P \text {. }
$$

Hence, by (5.4) and (5.5), we have

$$
\operatorname{dim} \widehat{B}=\operatorname{ht} m=\operatorname{ht}(M / P)+\operatorname{ht} P=\operatorname{ht} M \leq \operatorname{dim} \widehat{A} .
$$

Thus, $\phi$ is an isomorphism and we may identify $\widehat{B}$ with $\widehat{A}$.

Since $A$ is birational to $B, B_{m} \subseteq A_{M}$ and $\widehat{B}=\widehat{A}$, we have $A_{M} \hookrightarrow \widehat{A}$.

Now, since $\widehat{B}$ is faithfully flat over $B_{m}$, we have $B_{m}=\widehat{B} \cap \operatorname{qt}(B)$, where $\operatorname{qt}(B)$ denotes the field of fractions of $B$. Therefore, since $A_{M} \subseteq \widehat{A} \cap \operatorname{qt}(A), \widehat{B}=\widehat{A}$ and $\operatorname{qt}(B)=\operatorname{qt}(A)$, we have $A_{M}=B_{m}$. Therefore, $A_{M}$ is a locality over $R$, as desired. This completes the proof.

Acknowledgements. The second author acknowledges Department of Science and Technology for their SwarnaJayanti Fellowship.

\section{References}

[1] S.S. Abhyankar, P. Eakin and W. Heinzer, On the uniqueness of the coefficient ring in a polynomial ring, J. Algebra 23 (1972) 310-342. 
[2] S.M. Bhatwadekar and Neena Gupta, The structure of a Laurent polynomial fibration in $n$ variables, J. Algebra, 353 (1) (2012) 142-157.

[3] I.S. Cohen, Lengths of prime ideal chains, Amer. J. Math. 76 (1954) 654-668.

[4] A.K. Dutta and N. Onoda, On finite generation of $R$-subalgebras of $R[X]$, J. Algebra 320 (2008), 57-80.

[5] P. Eakin, A note on finite dimensional subrings of polynomial rings, Proc. Amer. Math. Soc. 31(1) (1972) 75-80.

[6] R.M. Fossum, The Divisor Class Group of a Krull Domain, Springer Ergebnisse $\mathbf{7 4}$ Springer-Verlag (1973).

[7] J.M. Giral, Krull dimension, transcendence degree and subalgebras of finitely generated algebras, Arch. Math. 36 (1981), 305-312.

[8] H. Matsumura, Commutative Algebra (Second Edition), Mathematics Lecture Note Series, Benjamin/Cummins Publishing Co. (1980).

[9] H. Matsumura, Commutative Ring Theory (reprint of first paperback edition with corrections), Cambridge University Press, Cambridge (1990).

[10] N. Onoda, Subrings of finitely generated rings over a pseudo-geometric ring, Japan. J. Math. 10(1) (1984), 29-53.

[11] N. Onoda, A theorem on subrings of affine rings and its application, Math. J. Toyama Univ. 20 (1997), 99-106.

[12] M. Nagata, Local Rings, Interscience Tracts pure appl. math. vol. 13, Interscience, New York, 1962.

[13] M. Nagata, A theorem on finite generation of a ring, Nagoya Math. J. 27 (1966), $193-205$.

[14] O. Zariski and P. Samuel, Commutative Algebra, vol. II, Van Nostrand, Princeton, 1960. 